\title{
NEUROPSYCHOPHARMACOLOGY REVIEWS Let's call the whole thing off: evaluating gender and sex differences in executive function
}

\author{
Nicola M. Grissom ${ }^{1}$ and Teresa M. Reyes ${ }^{2}$
}

The executive functions allow for purposeful, deliberate, and intentional interactions with the world-attention and focus, impulse control, decision making, and working memory. These measures have been correlated with academic outcomes and quality of life, and are impacted by deleterious environmental events throughout the life span, including gestational and early life insults. This review will address the topic of sex differences in executive function including a discussion of differences arising in response to developmental programming. Work on gender differences in human studies and sex differences in animal research will be reviewed. Overall, we find little support for significant gender or sex differences in executive function. An important variable that factors into the interpretation of potential sex differences include differing developmental trajectories. We conclude by discussing future directions for the field and a brief discussion of biological mechanisms.

Neuropsychopharmacology (2019) 44:1-11; https://doi.org/10.1038/s41386-018-0179-5

\section{INTRODUCTION}

Executive function refers to a collection of abilities that allows an individual to organize their behavior to respond to the current environment and plan for future situations. These skills, including attention, impulse control, decision making, and working memory, allow an organism to attend to relevant variables, compare these variables to past experiences, evaluate which responses would be most effective, and inhibit inappropriate responses. Welldeveloped executive functioning will contribute to an individual's success, socially, academically, and professionally. However, disruption of executive function is associated with adverse outcomes and is a component of numerous mental health disorders, including attention deficit hyperactivity disorder (ADHD), schizophrenia, and bipolar disorder.

However, it is well-known that the above named neuropsychiatric conditions are not typically matched between genders in diagnosis rates, onset, severity, and/or prognosis. For example, ADHD is more likely to be diagnosed in boys [1]. A meta-analysis revealed that impulsivity was significantly more prevalent in boys with ADHD versus girls, while no gender differences in attention were indicated [2]. Moreover, the presentation of ADHD may differ between the sexes, with impulsivity more often diagnosed in boys [3]; however, other studies have reported similar presentation of ADHD symptoms between the sexes [4-6]. Further, adult women with ADHD have more severe symptoms if they were diagnosed with ADHD as a child, while this relationship is not present in boys [7]. The incidence of schizophrenia is higher in males [8], and may be slightly more prevalent in men [9], and the initial presentation is different by sex, with onset typically seen later in females $[10,11]$. Bipolar disorder equally affects men and women, but again the onset and presentation differs, with onset occurring later in women, and women showing more rapid cycling between mania and depression than men [12]. These differences extend to work in animal models of these disorders as well [13, 14]. One question that emerges is whether fundamental sex and gender differences in the different executive functions exist as a possible mechanism contributing to the sex- and gender-disparate risk for neuropsychiatric conditions. In this review, we examine the evidence for baseline sex and gender differences in four essential executive functions in both the human and animal literatures.

Executive function can be impacted by deleterious events throughout the life span, including gestational and early life insults, stress, drug abuse, and aging [15-20]. These adverse environmental events are also linked to an increased risk for the development of many, if not most, neuropsychiatric conditions including mood disorders, dementias, psychosis, and neurodevelopmental disorders such as autism and attention deficit/ hyperactivity disorder. Therefore, the present review will approach the topic of sex differences in executive function with a particular focus on differences related to developmental programming. We conclude that (1) individual components of executive functions may be enhanced in one sex or gender over another, but that there is no systematic advantage, (2) that these effects depend greatly on the modality of testing and the parameters tested, suggesting that apparent differences in the abilities in executive functions may in fact reflect different strategies employed by each sex and gender when confronted with a challenge or ambiguous situation and (3) early life adversity can disrupt executive function, with important influences of both gender/sex and age/timing on the severity and expression of these effects.

\section{Human gender differences in attention}

Attention and impulsive action are often measured concurrently, within the same tests, and will therefore be discussed collectively here. A wide variety of neuropsychological tests are available, including the widely used continuous performance task (CPT).

\footnotetext{
'Department of Psychology, University of Minnesota, Minneapolis, MN 55455, USA and ${ }^{2}$ Department of Psychiatry and Behavioral Neurosciences, University of Cincinnati, Cincinnati, $\mathrm{OH} 45237$, USA

Correspondence: Teresa M. Reyes (reyesta@ucmail.uc.edu)
}

Received: 30 March 2018 Revised: 30 July 2018 Accepted: 6 August 2018

Published online: 14 August 2018 
While the specifics of the task can vary (type of stimulus presentation (visual vs. auditory) or the required response), the basic strategy of the CPT remains the same. In the CPT, individuals must respond correctly to target stimuli, and inhibit responding to incorrect stimuli (e.g., presentation of the letters $A$ and $B$, in which responding should occur only on the presentation of $A$ (target) and never to $B$ (foil)). If the target is presented repeatedly, in rapid succession, followed by a single presentation of the foil, it can be difficult to inhibit responding to the foil, which is a measure of impulsive action. Similarly, if the target is only presented very infrequently, the subject is required to pay close attention, and errors of omission are a measure of inattention.

Age of testing, as well as the specific testing modality, impact the findings of gender differences in attention. In a normative sample of 8- and 10-year-old children tested with a standard neuropsychological battery, girls were found to have better scores on attention as compared to boys [21]. However, testing later, during adolescence, using the CPT, revealed no gender differences in attention [22].

Another study that examined the full life span (ages 17-90) failed to find any gender differences in attention; however, women were found to have slower reaction times [23], consistent with another report using adults that reported slower reaction times in females, but no gender differences in attentional performance [24]. Another study that used responses in an online format of the CPT from a very large number of respondents $(n=$ 9-11,000/gender) also indicated a slower reaction time in women, as well as gender differences, such that females made more omission errors, while males made more commission errors (responding inaccurately to the foil); however, it was noted that the effect size of these differences was quite small [25].

Human gender differences in impulsive action

Analysis of typically developing 8- and 10-year-old children found that girls were less impulsive than boys [21]; however, by adolescence, girls scored higher in impulsive action in the CPT compared to boys [22]. Similar to the findings in adults, 9-10-yearold boys were found to have faster reaction time in a go-no/go task as compared to girls, a finding that was also seen in 9-17year-old population [26, 27]. In that population, boys were also found to be more impulsive [26]. The fact that boys may have faster reaction times may underlie or contribute to the noted increase in impulsive action, particularly when tested in CPT, as premature responses are more likely with a faster reaction time and are coded as impulsive behavior.

\section{Sex differences in animals in attention and impulsive action}

The five-choice serial reaction time task (5CSRTT) has been used to assess attention and impulsivity in mice [28], and is considered the gold standard for evaluating executive function deficits in rodents. This task is analogous to the CPT used to assess sustained and selective attention in humans [29], and screen for ADHD [30]. This operant task requires localization of brief visual stimuli presented randomly in one of five locations. A large number of correct target detections indicates good attentional performance as reflected by high response accuracy accompanied by few errors. The intertrial interval (ITI) can be manipulated to make the task more challenging. A longer ITI can increase premature responding, while a variable length ITI can challenge attentional systems, as an animal can no longer rely on timing strategies to indicate the presence of the cue. Assessment of higher executive function in rodents is relatively time and resource intensive, limiting the broad adoption of these assays in research. Consequently, there are limited studies overall, and of those, only very few that include females.

Attention. In one of the earliest studies to assess both male and female mice in the 5CSRTT, no sex differences were identified in acquisition of the task, or performance metrics (accuracy, omissions, premature responses); however, female mice were reported to have faster reaction times [31]. Importantly, this paper also reported how changes in task difficulty impacted performance. When the intertrial interval (ITI) was lengthened, impulsive errors increased acutely in both males and females, but males rapidly habituated, while female premature responding persisted. Similarly, in adult rats, females appear to be more adversely affected by increasing task difficulty. Females, more so than males, showed a greater decrement in attention under challenging $5 \mathrm{C}$ parameters, either a shorter stimulus and variable intertrial interval (ITI; [32] or when the ITI was long [33]). Environmental changes have also been shown to impact performance in a sex-dependent manner, with males showing an increase in susceptibility to performance decrements. Exposure to a mild stressor was found to decrease attention and increase impulsive responding in male mice, while female accuracy was not affected [31]. This group also tested animals that were ad lib fed (rodents are typically food restricted for the 5CSRTT to increase motivation to perform) to remove the stress and motivation factors. In this context, females outperformed males, and were more accurate and had fewer premature responses. Vitamin $D$ deficiency has also been examined in adult mice. Similar findings were observed here, as it was shown that males were the only sex affected, with vitamin D-deficient males showing an increase in reaction time, less accurate performance, and more inattentive errors in the 5CSRT, while there were no adverse effects of vitamin $D$ deficiency in females [34].

Impulsive action. To examine impulsive action in rodents, the 5CSRTT is used. In our work, we did not observe any sex differences in attention, but did note a higher rate of impulsive errors in adult females versus male mice under baseline task conditions [35]. Similarly, using a modified version of the 5CSRTT to allow for testing in younger animals, adolescent female mice were found to make more impulsive errors [36]. Reaction time has also been examined, and no sex differences have been reported $[33,35,37]$. When parameters are changed to make the task more difficult (shorter stimulus and variable intertrial interval), males have shown increased impulsive action [32]. Similarly, in another study, males made more premature responses (impulsive action) when the ITI was long [33]. Additionally, male rats were found to be more impacted by a variable ITI, leading to more impulsive errors in 5CSRTT, while female performance remained robust, even in the face of increasing task difficulty [38]. However, another study using a two-choice task found that with increasing difficulty (long ITI), in adolescence, males were more impulsive than females, while as adults, females made more premature responses as compared to males [37]. Clearly, there are important developmental differences with regard to impulsive behavior, as it has also been reported that male rats showed greater preference for novelty and increased activity earlier in life, whereas these behaviors emerged later, during adolescence, in females [39].

Overall, considering both human and animal studies, one can conclude that large gender or sex differences in attention and impulsive action are absent. Baseline differences in attentional performance are not described; however, the extent to which subjects are adversely affected by task difficulty or environmental stressors differs by gender/sex, such that females appear to be more adversely affected by task difficulty and males by environmental perturbations. There may be support for an increase in impulsive action in males, potentially related to a faster reaction time seen in human studies. In animals, a few studies support increased impulsive action (at baseline) in female animals; however, males appear to be more impacted by increases in task difficulty or changes in the testing environment. 
Decision making and impulsive choice

Aberrant decision-making processes are seen in a variety of neuropsychiatric conditions, including autism, psychosis, mood disorders, and addictions, reflecting both vulnerabilities in the ability to use this executive function, and in many cases contributing to worsening outcomes.

The process of decision making involves two key components: the ability to determine the most likely outcomes from a given choice, and the ability to weigh different choices according to probable outcomes and select an optimal choice. We can think of these processes as first, establishing the rewards and risks of various choices through learning when making decisions in unfamiliar circumstances, often measured in the early phases of decision-making tasks, and second, making selections when substantial evidence has been collected about relative rewards and risks among various options, often measured later in decisionmaking tasks.

Therefore, the potential role for sex and gender differences to influence decision-making processes could occur during the early stages of evidence collection and learning, or later, through differential weighting of risk and reward between sexes and genders. However, evidence collection processes are likely to be heavily influenced by rates of risk and reward. In fact, as discussed below, a differential sensitivity to risk versus reward, and the balance of risk versus reward in a given task, shifts the influence of sex and gender in decision-making tasks. While there is an apparent gender/sex effect in decision making, this appears to be driven by sex differences in the assessment of risk, not rewards, causing females to avoid frequent loss even if this is covertly advantageous, especially during learning in unfamiliar circumstances [40, 41]. When a task is run where loss frequency is controlled, or once sufficient evidence has been collected about risks and rewards, males and females are equally adept at making optimal decisions.

\section{Human gender differences in decision making and impulsive} choice

The most frequently employed decision-making task in humans is the lowa Gambling Task (IGT), which was originally developed several decades ago as a method to examine the ability of losses to drive shifts in decision making in neuropsychological patients through long-term estimations of value. The ubiquity of this task means that there is quite a bit of data on the role of gender and sex, but confounds in the design $[42,43]$ mean that the interpretation of these data becomes challenging. At first glance, there appear to be differences in performance between genders on this task. However, careful analyses have shown that these are driven by gender differences in tolerance for frequency of loss rather than magnitude of gains, which are confounded.

Briefly, the IGT involves four decks of cards, each of which has cards that gain money, and cards that lose money. Over the long term, two of these decks ( $A$ and B) have a net loss of $\$ 250$ over every ten cards, and two of these decks ( $C$ and $D)$ have a net gain of $\$ 250$ over ten cards. However, the decks differ not only in their "final outcome", but in the probability of choosing a losing card on any turn; decks $A$ and $C$ have a rate of five losses and five gains over every ten cards, while $B$ and $D$ have a rate of one loss and nine gains over every ten cards. Finally, decks $C$ and $D$ have cards that have smaller individual gains ( $\$ 50$ per card) versus decks $A$ and $B(\$ 100$ per card); thus, there is no deck with both large individual gains and a beneficial final outcome. Thus, for ideal performance, participants have to make a counterintuitive decision to forgo large gains (which are frequent in deck B) in favor of smaller gains (deck $C$ ) and often to select more frequent losses (as in deck D). Participants in these experiments usually have 100 chances to pick a card from any of the decks.

A number of studies have shown that men tend to outperform women at the IGT, specifically by choosing from decks $C$ and D more frequently [42, 44-47]. However, many studies have failed to find any gender difference in this task [42, 48, 49], suggesting this effect is modulated by environmental factors to which men and women may be differentially susceptible and that are not typically controlled for between genders, such as stress and anxiety $[46,50-53]$. Performance on this task is often rated by the number of "gain" cards from the $C$ and $D$ decks a participant collected at the end of 100 trials. Critically, both men and women prefer deck $D$ with a net gain over time and only one loss per ten cards, over the other three decks. However, men tend to develop a preference for a deck (C) with frequent losses of smaller magnitude, while women maintain a preference for a deck with rare losses, although of large magnitude (B) [42]. Given additional trials past 100 , women develop a preference for deck $C[42,44]$, suggesting that women weight the frequency of loss more highly than men, while men rate the magnitude of loss more highly, when these options are put into conflict.

Because the IGT varies both frequency of loss and magnitude of loss, avoiding frequent loss can mean enduring losses of higher magnitude. However, it is possible to vary the frequency of loss while holding the magnitude of loss constant. In scenarios such as this, we would expect that this would lead to no sex differences in learning to make optimal choices, or even show enhanced performance in women by avoiding frequent loss. There is support for both of these possibilities in tasks where only loss frequency or loss magnitude are varied and the other option is controlled. In a modified version of the IGT, when men and women are given a choice only between decks $A$ and $C$, which both have frequent losses, people of both sexes prefer deck $C$, with a net gain. However, when the task is modified to contain only decks $B$ and $D$, which both have rare losses, people fail to distinguish that $B$ leads to a greater magnitude of loss than $D$ and choose them at equivalent rates [54, 55]. This suggests that observed gender differences in decision making as measured by the IGT are driven by wishing to avoid frequent loss, not by a gender difference in the ability to detect loss magnitude.

Another kind of decision-making task that varies only the frequency of loss/reward while keeping the magnitude of these outcomes constant is probabilistic decision making, sometimes called a multiarmed bandit task. These kinds of tasks provide two or more items which vary in the probability of reward, but provide the same size of reward across options. These kinds of tasks have been recently used to examine decision-making strategies, particularly whether a participant chooses to explore unknown options versus choosing to exploit options with which they have prior experience. Studies in humans have largely found no gender difference in bandit task performance [56-58], suggesting that the ability to identify items with a high probability of reward does not vary as a function of gender. When the learning phase of this kind of task was specifically examined, one study found that women were quicker to learn to choose an option associated with a high probability of reward [57]. A different probabilistic repeated gambling task where there was no learning component (deciding whether a number was likely to be higher or lower than a provided number) found no differences in choices as a function of gender [59]. This suggests that observations that women avoid frequent losses in the IGT may be the other side of the coin wherein men are willing to make choices associated with a higher probability of loss, even when loss is highly probable.

A final form of decision-making task varies the magnitude of outcomes and varies the cost of the high-magnitude option, either as temporal delay (delay discounting) or in probability of occurrence (probability discounting, also called a risky decisionmaking task). These kinds of tasks typically ask whether a person would prefer an immediate and guaranteed small reward, versus a larger reward at some delay, or with some chance of occurring. These kinds of discounting tasks have generally failed to show baseline gender differences in the tendency to prefer an 
immediate reward [60-62], though gender can interact with other conditions such as alcoholism to result in gender-specific effects [60]. Fewer studies have been conducted with probability discounting in humans with gender-specific analysis, but these have generally not seen evidence for gender differences in healthy controls $[62,63]$.

Overall, these findings support the idea that there is little gender difference in decision making in general. However, this is dependent on a task design that controls for the frequency of gains and losses, compared to the magnitude of these events, as the avoidance of frequent losses would lead to the observation of decreased performance in the IGT and increased performance in bandit tasks, as described above. These findings are consistent with a broader literature indicating greater sensitivity of women and female animals to both rewarding and punishing outcomes, indicating that while decision making itself is not consistently different between genders, the information used to influence decisions may be processed differently by men and women.

Animal sex differences in decision making

The subject of sex differences in animal models of decision making was recently beautifully reviewed by Orsini and Setlow [41]. Here, we will discuss this literature with an emphasis on drawing connections to the human findings. Briefly, several different gambling-type tasks have been developed for testing in animal models in operant chambers, and again, the relationship between the magnitude of negative outcomes versus the frequency of negative outcomes [64] appear to determine whether there is a female advantage or a male advantage. A clear finding across animal tasks tested so far is that females will avoid making choices associated with frequent negative outcomes, such as shock or unpalatable food, even if these options are also associated with higher magnitude positive outcomes (more palatable food) [41]. However, in tasks where options differ only in the cost or probability of a positive outcome, without punishment, there is either no sex difference or a female advantage.

As in human tasks, animal tasks can include an overt negative outcome, and as in human literature, these tasks lead to females avoiding frequent negative outcomes. A rodent version of the IGT compared choices in an operant chamber for a long-term advantageous option (with small amounts of palatable pellets and occasional highly unpalatable pellets), and a disadvantageous option (with large amounts of palatable pellets but more frequent punishment by delivery of highly unpalatable pellets). Performance at the end of this task did not differ between sexes, but males learned to stay with the advantageous option more quickly, despite punishment. Females, in contrast, were more likely to shift to the other option following punishment, suggesting that the avoidance of deleterious outcomes was more motivating [65]. Paralleling this, Orsini and colleagues recently employed a rodent risky decision-making task where animals were asked to choose between an option that resulted in a small food reward, and an option that resulted in a larger food reward paired with some probability of footshock. Females showed a strong preference for the safe option, although smaller, than the risk of footshock [66]. These findings are consistent with those from the human literature indicating that females weight deleterious outcomes more highly in decision making.

The vast majority of tasks evaluating decision making in rodent models at this time involve active punishment to make a highmagnitude reward less attractive. As noted above, the human literature indicates that tasks which set up a conflict between the ability to detect profitable options with the desire to avoid frequent negative outcomes lead to an apparent deficit in female decision making. Because of this, it is helpful to compare the findings from the rodent version of the IGT to a task that changes the probability of negative outcomes while holding these at the same magnitude. The "rat Gambling Task", in contrast to the tasks described above, is more similar to a "bandit-style" task, in that losses involve the omission of a food reward, rather than an actively deleterious outcome such as unpalatable food or a shock. In addition, it involves forced choices, where females and males are obligated to pick each of the options in isolation, before being allowed to choose. Here, females learn more quickly than males to choose the advantageous option [67].

In delay discounting tasks, where animals choose between a small immediate food reward, and a larger food reward that occurs on a variable delay, there have largely been no significant sex differences reported [68-70], suggesting again that without active aversive outcomes, intact females and males assess reward outcomes similarly or with a slight tendency in females towards the detection of positive outcomes. Further, developmental time is a critical lens through which sex differences should be viewed. In a study that tested animals at early adolescence, late adolescence, and adulthood, an interesting pattern of sex differences was noted, and this related more to the developmental pattern of impulsive choice, as opposed to a simple sex main effect. In females, an inverted U-shaped curve was identified with impulsive choice peaking at late adolescence; however, in males the curve was quite different, remaining relatively high throughout adolescence, and decreasing only in adulthood [39].

Overall, as in the human data, animal decision-making research suggests that there is not a clear-cut sex difference or advantage, but a difference in outcome sensitivity that, critically, benefits males when punishments are severe and benefits females when punishments are minimal. Thus, again, while decision making itself does not appear to differ between animals of different sexes, the information processing that supports decision making does.

\section{WORKING MEMORY}

Working memory performance is the ability to maintain in mind a particular set of information, particularly a sequence of stimuli or events, for use in the immediate future to either repeat this sequence (for example, to maintain a trace of what the current topic of conversation is) or to avoid it (for example, to avoid rechecking a location that was just searched when looking for an object). As such, it is essential for normal cognitive and social function. Neuropsychiatric conditions, particularly schizophrenia, are often associated with profound working memory impairments.

As described above, working memory appears to be a unitary construct applied to any sequence of information, be it spatial, verbal, or associated with some other grouping of stimuli. However, these stimulus domains are supported by significantly different neural circuits, which are known to show sex and genderdisparate function-for example, hippocampal and temporal lobe circuits in processing spatial information versus cortical regions important to verbal, speech, and language processing. It transpires that the type of information that a human or animal is asked to maintain in working memory appears to influence the direction of observed sex differences-however, in large part there appear to be no sex differences in working memory function.

\section{Human assessment of working memory}

Working memory tasks can ask a person to keep in mind, for a short period of time, an item or series of items, such as a sequence of letters or numbers in n-back tasks, a particular object in delayed match to sample/delayed nonmatch to sample tasks, or a set of recently visited or noted spatial locations, as in object location tasks or human analogs of the radial arm maze.

Tasks designed to measure working memory have largely failed to identify any sex differences across a wide developmental range, from young children to elderly populations, though there are significant age-related changes in this ability. Young children show no sex differences in spatial working memory [71-73], and 
the same is true for adolescents [74], young adults [75-77], and aged adults [78]. When a gender disparity in working memory ability has been seen, women outperform men, particularly in spatial and location-based tasks [79-81], though this may differ at very high working memory loads [82]. A recent meta-analysis explicitly directed at identifying potential sex differences in working memory affirmed a female advantage in object location memory and suggested that there may be a male advantage in $n$ back tasks [83]. However, this analysis was limited to studies directly listing sex differences as a dependent measure, which reduced the sample from 2072 relevant papers to 69 , suggesting a possible influence of publication bias for papers identifying a sex difference and removing ones that did not observe differences that justified doing an exploratory sex differences analysis, despite the best attempt of the authors of this meta-analysis to avoid this. Further, this study identified a sex difference not in working memory as a general construct, but in specific domains in which working memory is applied.

One potential mechanism that could produce a slight tendency for sex- and gender-disparate performance in different working memory tasks is the existence of sex-specific strategies in working memory. In a spatial working memory task in adolescents where there were no sex differences detected in successful performance, there were nevertheless substantial differences in patterns of neural activity as measured by fMRI [74], such that girls showed reduced activation of the default mode network, while boys showed activation in regions previously associated with spatial working memory function. This finding suggests that either there may not be a single canonical network necessary for working memory, or that the baseline activity of the default mode network versus task-focused networks may differ between sexes. Altering the types of information available in spatial working memory tasks reveals gender-specific behavioral strategies such that women appear to rely on allocentric cues to direct behavior, whereas removal of global spatial or directional cues impairs performance in men [84]. This finding helps to resolve discrepancies with the animal literature, discussed below, that has tended to find a male advantage in working memory tasks, and suggests that the trend towards a female advantage in human working memory tasks may be related to the availability of allocentric information in a given task as conducted in the laboratory.

\section{Animal assessments of working memory}

The assessment of working memory in rodents has often relied on spatial working memory, particularly the radial arm maze task. As discussed below, this task has often, but not always, identified a male advantage in the task, which is in conflict with the general trend of no sex difference or a female advantage in working memory assessed in humans. The magnitude of differences in performance in the radial arm maze is not large, and idiosyncrasies in the way the task is typically assessed and reported may drive this apparent discrepancy between the human and animal literatures. In rodents and nonhuman primates, a number of object-based tasks, including delayed match to sample, delayed nonmatch to sample, novel object recognition, and spatial object recognition, have largely identified no sex difference in performance.

The radial arm maze task employs a number of arms (typically 8 or 12) arranged like spokes from a center hub, and some or all of these arms may be baited with a small food reward. The most typical measure for this task is the total number of arm entries to collect all the available food. Males have repeatedly been shown to use a lower number of total arm entries to complete this task [85-89], but there have also been a number of papers showing no sex difference in this measure $[90,91]$. This result has often been interpreted to mean that males are better at maintaining in mind which arms have already been visited, and thus that males have an advantage in working memory in general. However, there are a number of caveats to this interpretation. First, the size of the maze relative to a rodent may shift the preponderance of available cues away from allocentric cues and towards global spatial cues, and thus the kind of information that females use preferentially in working memory may be less available. Second, the literature on decision making discussed above revealed a female bias in the avoidance of negative outcomes, suggesting that at some point in running the maze, females may prioritize checking to ensure a food reward has been collected from a previously visited arm rather than risk losing out on that food reward. Several pieces of evidence suggest that female rodents engage in exactly this strategy when solving the radial arm maze. First, in a study which did not find an overall sex difference in the total number of arm entries, it was found that females engaged in a higher degree of "vicarious trial-and-error", pausing and investigating arms with head entries before moving on to another arm, suggesting that females were investing more effort into determining whether or not a food reward had been collected at a given arm than males [90]. Second, a study in mice found that although females had higher total number of arm entries at the end of the maze, the number of arm entries before an arm was repeated was identical between sexes [88]. This latter measure is much less frequently reported, but is likely more directly related to a true working memory construct as it reflects the maximum number or "span" of arms that can be visited before an animal makes a first error. The overall error rate in radial arm maze testing, then, may reflect increased reliance in males on a behavioral strategy that may result the loss of a possible food reward, consistent with the decision-making literature.

Other tasks used in rodents and nonhuman primates that do not rely on the collection of multiple food rewards have found either no evidence of a sex difference or a trend towards a female advantage. These tasks involve the ability to hold in mind a given item (object, smell), and then determine whether it is the same (delayed match to sample), is different (novel object recognition), or has moved location (spatial object recognition). Note that the latter two tasks have both a long-term memory version that is tested over multiple days, or can be tested in a short period of time in a single day to assess working memory. Versions of these tasks where working memory was assessed found that female rodents were advantaged at recognizing a novel object [92] and at an olfactory delayed match to sample task [93]. Delayed match to sample tasks are frequently employed in nonhuman primates, but limitations in the numbers of animals used in a given experiment often limit the ability to investigate sex differences. However, two studies, one examining baboons [94] and one examining both chimpanzees and rhesus macaques [95] both failed to detect any sex difference in the performance of delayed match to sample. Overall, data from these object recognition tasks, combined with the human literature, suggest that there is not a strong sex difference in working memory, and to the extent that there is, this favors females, indicating that the male enhancement in radial arm maze performance is probably due to nonworking memory mechanisms such as sex differences in decision making.

\section{Programming}

We have now described how specific executive function components are evaluated in humans and animals, and reviewed the evidence for sex differences in these endpoints. While there is limited evidence to support baseline differences in executive function abilities, there is some support for gender/sex differences in the cognitive strategies used during testing as well as how different challenges, either within the task or as part of the environment, affect performance. As mentioned in the introduction, deficits in executive function are a component of numerous mental health disorders, including schizophrenia, bipolar disorder, ADHD, and autism. Interestingly, the etiology of each of these disorders is known to have a developmental component, and to 
be influenced to a greater or lesser extent by environmental stressors. Therefore, in the following section, we will discuss work that has examined how executive function deficits arise from adverse events in early life, and how these programming events may interact with gender/sex to differentially impact executive function outcomes.

Developmental processes in early life can substantively affect executive function. Deleterious conditions can lead to broadly ranging cognitive deficits that surpass the influence of sex $[35,73$, $96,97]$. Longitudinal studies in China and India, where substantial differences in early life conditions occur (often on a gendered basis), reveal that early life education alters whether men or women outperform each other in cognitive tasks [98-100] and that height is independently predictive of cognitive performance [101]. Because height is an indicator of the quality of gestational and early life conditions, these studies suggest that the evaluation of executive function in humans should control for educational attainment, parental investment, and factors that reflect quality of early life. A number of environmental exposures occurring either during prenatal or early postnatal development have been shown to affect executive function, including dietary changes, and exposure to toxins, infection/inflammation or glucocorticoids (GCs) being the most well-studied. For health outcomes in general, an emerging theme suggests that male offspring tend to be more adversely affected by early life adversity than females [102-106], an observation documented in both humans and animal models. To what extent this applies to executive function shall be discussed here.

Glucocorticoids are routinely given to babies in utero when there is imminent risk for a preterm delivery, in an effort to accelerate lung development. However, this treatment is not without adverse effects. In one study that examined children given the synthetic GC dexamethasone, exposed girls had increased inattention at ages 7-10, compared to a reference population, while boys did not show any increase in negative effects [107]. Similarly, in a study using baboons, female baboons made more errors in discrimination and reversal learning tasks after prenatal exposure to dexamethasone, while males were not affected [108]. Therefore, with regard to the effects of early GC exposure on executive function, girls appear to be more adversely affected.

Maternal diet during pregnancy can also adversely affect the development of executive function in offspring. Using the 5CSRTT, we have shown that offspring from mouse dams fed a diet deficient in protein, a model of low birth weight at term, showed an increase in inattention, while offspring from dams fed a high fat diet, a model of excessive gestational weight gain, showed increased impulsivity [35]. Interestingly, these effects did not differ across the sexes, with both male and female offspring showing similar levels of errors. In another model of in utero growth restriction (maternal nutrient restriction of $70 \%$ of ad lib fed animals through gestation and lactation) in baboons, attentional deficits and impulsivity were detected in males but not females [109]; however, the samples sizes were quite small $(n=3-4 /$ group), and this study was likely underpowered to adequately assess sex differences.

An investigation into prenatal drug use found that methamphetamine or methylphenidate given to dams during pregnancy affected the offspring, with both drugs increasing impulsive action in the offspring, with only very subtle sex differences observed [110]. Similarly, in an animal model used to examine autismrelated endophenotypes, valproic acid administration to pregnant dams increased inattention, with no effect on impulsivity, in both male and female offspring, with very minor task-dependent sex differences noted [38].

Other maternal and/or pregnancy related-challenges have been examined, as well. A complicated delivery was associated with greater risk for ADHD diagnosis, and the effect size was bigger in boys than girls [111]. Maternal smoking has also been shown to increase the risk for ADHD, and at least one study found this risk did not vary by gender [112]. Environmental exposures to toxicants have been examined as well. Prenatal urinary concentrations of phthalate metabolites were found to be associated with inattention and behavioral difficulties in boys, but not girls [113]. Mild traumatic brain (mTBI) injury in rats was also found to have complex effects on executive function that varied by sex. mTBI led to more impulsive errors in the 5CSRTT in control males; however, more impulsive errors were identified only in females when examining offspring from nutrient-restricted dams [114], indicating that early life environment can also establish a sexdependent risk profile for future insults.

The literature on early life adversity and programming of working memory and/or decision making is extremely limited, even more so, with regard to sex/gender differences. Exposure to the glucocorticoid dexamethasone during the first trimester adversely affected spatial working memory only in girls, but not in boys [115], while prenatal exposure to pesticide (at very low levels) was associated with decreased neuropsychological scores in girls, but not boys aged 6-11 years [116]. With regard to decision making, no sex difference was observed using the rodent gambling task in rats, either in control animals or those exposed to vitamin D deficiency [67], and similarly, in a study that used females only, there was no effect of early life stress (maternal deprivation) on performance in the rodent gambling task [117].

Potential biological mechanisms contributing to gender/sex differences

Given the diversity of behaviors that are incorporated by executive function, there are a number of brain regions that have been linked to these varied endpoints. However, it is also worth noting that much of this work was largely or exclusively conducted in males, and may therefore fail to identify circuitry or neurochemistry unique to females (see below), particularly given the male -female differences in executive function strategies we have previously described. The prefrontal cortex has received significant attention with regard to executive function, with links to attention, impulsivity, and working memory, including both medial PFC (mPFC) [118-120] and the orbitofrontal cortex (oPFC) $[121,122]$. Normal PFC function requires a balance between excitatory drive from glutamate neurons and inhibitory drive from GABA neurons, and both GABA [120, 123, 124] and glutamate $[124,125]$ have been linked to executive function. Given the breadth of behaviors that contribute to executive function, the associated neurochemistry is correspondingly broad [126], indicating roles for dopamine and opioids [118, 127, 128], and acetylcholine [129], in addition to GABA and glutamate. Relevant to understanding sex/gender differences in executive function is the fact that sex differences in these key neurotransmitter systems, specifically within brain regions important in executive function (prefrontal cortex, striatum) have been observed. Basal sex differences in serotonin and dopamine were noted in the PFC of adolescent and adult rats [130], while sex differences in nicotinic receptor responses within PFC have been noted in rodents early in development [131]. Sex differences in dopamine receptor expression within striatum have also been documented [132]. Importantly, interesting genotype $\times$ sex interactions have been noted for two dopamine-related genes (COMT and DAT1) in a population cohort, such that COMT and DAT1 alleles associated with ADHD-related symptoms (inattention and hyperactivity) and a full ADHD diagnosis in boys, but there was no association in girls [133]. In animals, adolescent treatment with fluoxetine (a selective serotonin reuptake inhibitor) improved attention in females, but led to impairments in males, indicating that the effect of serotonin on attentional performance varies by sex [134].

Additionally, differences in structural brain development may contribute to sex differences in the programming of executive 


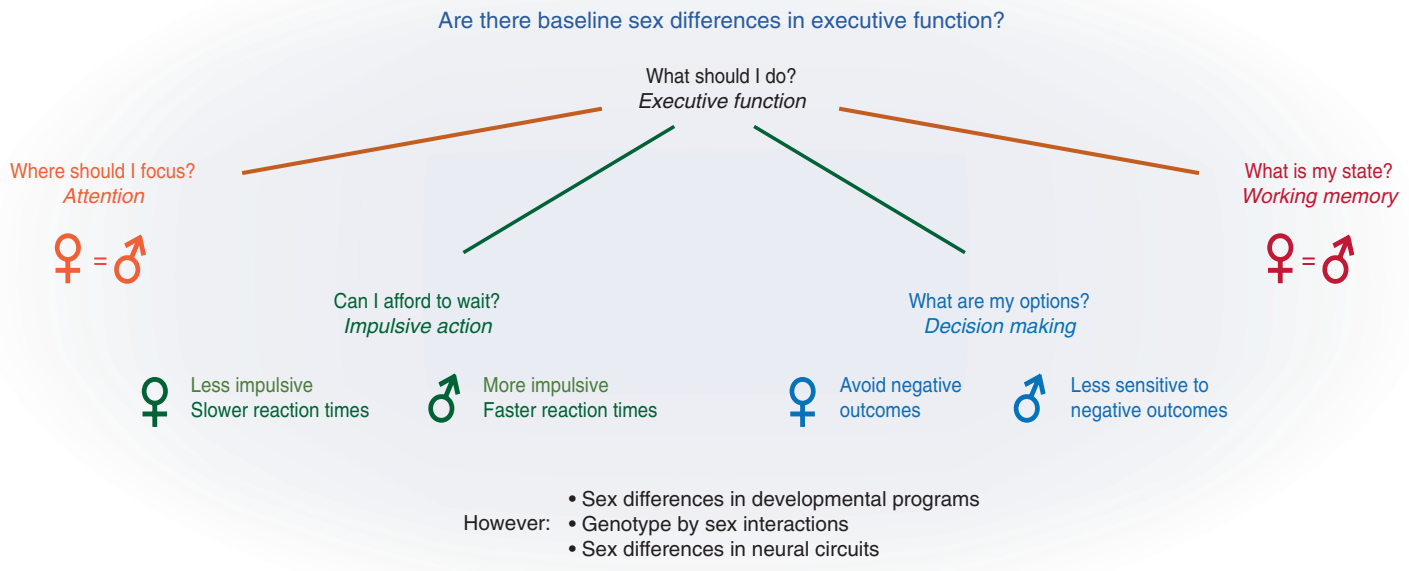

Fig. 1 Summary of sex and gender differences in executive function. Literature review supports the finding that there are small and subtle to no sex and gender differences in executive function. However, sex differences do emerge in the context of developmental programming, the interaction with genotype, and potential underlying biological differences

function. Brain development in early life involves the overproduction of neurons and their connections, which are later sculpted by neural activity, so that weakly active connections are removed during a process called "pruning" [135]. Overproduction of dendritic spines (a marker for excitatory synapses) is greatest in the PFC, and synaptic pruning occurs most slowly in the PFC, continuing well into the postnatal period. During synaptic pruning, "weak" synapses are eliminated, while functional synapses are strengthened. In the PFC, it is primarily excitatory synapses that are targeted for elimination [136]. Synaptic pruning is a key developmental process, as deficits in synaptic function are linked to neurodevelopmental disorders $[137,138]$ and executive function deficits [114]. Further, executive function deficits have been suggested to relate to more subtle changes in neural function or microstructure rather than gross changes in neuron number or morphology [138]. Further, the very fact that PFC development continues well into postnatal development (into the 20 s for humans) renders this brain region uniquely vulnerable to environmental perturbations, during pregnancy, early life, and into adolescence. Importantly, there are documented gender differences in cortical maturation [8] and complexity [139], particularly through adolescence.

As gender/sex differences in brain maturation are apparent, one can then question whether gender/sex differences can be identified in the cellular or molecular mechanisms that contribute to cortical maturation. A critical role for microglia and astrocytes in the process of synaptic pruning has been identified, specifically involving the complement system [140]. Complement proteins, $\mathrm{C} 1 \mathrm{q}$ and $\mathrm{C} 3$, are expressed on weak synapses that are then targeted for phagocytosis and clearance by microglia, a process first identified in the developing visual system [140]. Sex differences in this process are unknown; however, given the importance of microglia in synaptic pruning, one can speculate that sex differences in microglial numbers or function may alter the process of synaptic pruning in a sex-dependent manner. And in fact, sex differences in microglia have been recently characterized. The developmental trajectory of microglial population within the brain differs by sex [141, 142], and early postnatal removal of microglia leads to both sex-dependent and sex-independent behavioral changes [142]. Further, microglial responses to environmental insults are also known to differ by sex [143], including responses to acute or chronic stress [144, 145] or alcohol exposure [146].

\section{FUTURE RESEARCH DIRECTIONS}

In this review, we examined the evidence for baseline sex and gender differences in executive functions. Our overall conclusion is that while individual factors may show a tendency towards a sex bias (e.g., increased impulsive action in males, reduced reaction time in males, avoidance of frequent punishment in females, improved working memory in females), sex differences in executive function are not overwhelming (Fig. 1). Within-sex variability often far exceeds between-sex variability, and in few cases could one look at a given person or animal's data in these experiments (where provided) and be able to classify them by their responses as male or female. The fact that many studies draw different conclusions on whether there is a gender/sex difference in executive functions based on small modifications to task design suggest that differences in strategy and outcome preference drive apparent effects on executive function, rather than a difference in ability between genders/sexes. It would be incorrect to conclude that gender and sex is the primary factor driving individual differences in executive function and cognitive performance.

With this in mind, two critical future directions present themselves. First, the sex differences in strategy suggest that different circuit and/or molecular mechanisms are utilized by males and females to solve the same cognitive problems. This means that even though ability may be the same, the strategies employed are unlikely to be supported by the same neurobiological mechanisms. Indeed, investigations of male and female cognitive performance have identified sex-specific signaling patterns that suggest sex differences at a molecular level driving apparently similar cognitive performance in wild types, but differential susceptibility to a genotype associated with autism and neurodevelopmental disorders [147]. Thus, it is critical that females and males of all species continue to be examined, to determine the mechanisms supporting executive function across genders and sexes. Indeed, this work may identify alternative pathways that can be harnessed and enhanced in patients with disorders associated with executive function deficits, as literature suggests that there may be sexspecific circuit engagement [148-151]. 
An additional critical future research direction is implied by the fact that disorders with large sex and gender differences in presentation often occur at different times of life. For example, neurodevelopmental disorders such as ADHD and autism tend to affect boys more frequently, while mood disorders appearing in adolescence and young adulthood are more frequently noted in girls and women. Further, schizophrenia diagnoses in males peak between 46 and 55 years of age, while in females the peak of diagnoses occur later, between ages 56 and 65, and at an early timepoint (age 18-29), the prevalence rate is more than double in males [139]. These observations suggest that sex and gender interact with developmental processes to influence specific deficits in executive functions in specific disorders. For example, while working memory processes are largely similar between healthy women and men, women with Obsessive-Compulsive Disorder (OCD) tend to have more severe working memory deficits than men with OCD [77]. Neurodegenerative and brain injury effects on executive function may also reflect different risks for men versus women [76]. Future studies should expect that sex and gender interact with their causal mechanism of interest, and specifically examine how executive functions are impacted differently in men and women, or male and female animals, by specific genotypes and environments.

\section{ACKNOWLEDGEMENTS}

Support was provided by NIH MH106330 (TMR) and the Simons Foundation Autism Research Initiative (NMG) and the Klarman Family Foundation (NMG).

\section{ADDITIONAL INFORMATION}

Competing interests: The authors declare no competing interests.

Publisher's note: Springer Nature remains neutral with regard to jurisdictional claims in published maps and institutional affiliations.

\section{REFERENCES}

1. Morgan PL, Staff J, Hillemeier MM, Farkas G, Maczuga S. Racial and ethnic disparities in ADHD diagnosis from kindergarten to eighth grade. Pediatrics. 2013;132:85-93.

2. Hasson R, Fine JG. Gender differences among children with ADHD on continuous performance tests: a meta-analytic review. J Atten Disord. 2012;16:190-8

3. Newcorn JH, Halperin JM, Jensen PS, Abikoff HB, Arnold LE, Cantwell DP, et al. Symptom profiles in children with ADHD: effects of comorbidity and gender. J Am Acad Child Adolesc Psychiatry. 2001;40:137-46.

4. Gunther T, Knospe EL, Herpertz-Dahlmann B, Konrad K. Sex differences in attentional performance in a clinical sample with ADHD of the combined subtype. J Atten Disord. 2015;19:764-70.

5. Novik TS, Hervas A, Ralston SJ, Dalsgaard S, Rodrigues Pereira R, Lorenzo MJ, et al. Influence of gender on attention-deficit/hyperactivity disorder in EuropeADORE. Eur Child Adolesc Psychiatry. 2006;15:115-24.

6. Omura K, Kusumoto K. Sex differences in neurophysiological responses are modulated by attentional aspects of impulse control. Brain Cogn. 2015;100:49-59.

7. Millenet $S$, Laucht $M$, Hohm $E$, Jennen-Steinmetz $C$, Hohmann $S$, Schmidt $M H$ et al. Sex-specific trajectories of ADHD symptoms from adolescence to young adulthood. Eur Child Adolesc Psychiatry. 2018; 8:1067-1075.

8. McGrath J, Saha S, Welham J, El Saadi O, MacCauley C, Chant D. A systematic review of the incidence of schizophrenia: the distribution of rates and the influence of sex, urbanicity, migrant status and methodology. BMC Med. 2004;2:13.

9. Kessler RC, Birnbaum H, Demler O, Falloon IR, Gagnon E, Guyer M, et al. The prevalence and correlates of nonaffective psychosis in the National Comorbidity Survey Replication (NCS-R). Biol Psychiatry. 2005;58:668-76.

10. Goldstein JM, Cherkerzian S, Tsuang MT, Petryshen TL. Sex differences in the genetic risk for schizophrenia: history of the evidence for sex-specific and sexdependent effects. Am J Med Genet B Neuropsychiatr Genet. 2013;162B:698-710.

11. Hafner $\mathrm{H}$, an der Heiden W. Epidemiology of schizophrenia. Can J Psychiatry. 1997;42:139-51.
12. Arnold LM. Gender differences in bipolar disorder. Psychiatr Clin North Am. 2003;26:595-620.

13. Hill RA. Sex differences in animal models of schizophrenia shed light on the underlying pathophysiology. Neurosci Biobehav Rev. 2016;67:41-56.

14. Kokras N, Dalla C. Sex differences in animal models of psychiatric disorders. Br J Pharmacol. 2014;171:4595-619.

15. Sanchez MM, Hearn EF, Do D, Rilling JK, Herndon JG. Differential rearing affects corpus callosum size and cognitive function of rhesus monkeys. Brain Res. 1998;812:38-49.

16. Bolandzadeh N, Davis JC, Tam R, Handy TC, Liu-Ambrose T. The association between cognitive function and white matter lesion location in older adults: a systematic review. BMC Neurol. 2012;12:126.

17. Girotti M, Adler SM, Bulin SE, Fucich EA, Paredes D, Morilak DA. Prefrontal cortex executive processes affected by stress in health and disease. Prog Neuropsychopharmacol Biol Psychiatry. 2017; 85:161-179.

18. Hostinar CE, Stellern SA, Schaefer C, Carlson SM, Gunnar MR. Associations between early life adversity and executive function in children adopted internationally from orphanages. Proc Natl Acad Sci USA. 2012;109: $17208-12$.

19. Kim-Spoon J, Kahn RE, Lauharatanahirun N, Deater-Deckard K, Bickel WK, Chiu $\mathrm{PH}$, et al. Executive functioning and substance use in adolescence: Neurobiological and behavioral perspectives. Neuropsychologia. 2017;100:79-92.

20. Lovic V, Fleming AS. Artificially-reared female rats show reduced prepulse inhibition and deficits in the attentional set shifting task--reversal of effects with maternal-like licking stimulation. Behav Brain Res. 2004;148:209-19.

21. Barnett JH, Heron J, Ring SM, Golding J, Goldman D, Xu K, et al. Gender-specific effects of the catechol-O-methyltransferase Val108/158Met polymorphism on cognitive function in children. Am J Psychiatry. 2007;164:142-9.

22. Lange K, Thamotharan S, Sferra M, Ramos A, Fields S. Effects of weight and gender on a task of inattention. Eat Behav. 2014;15:574-7.

23. Giambra LM, Quilter RE. Sex differences in sustained attention across the adult life span. J Appl Psychol. 1989;74:91-5.

24. Pletzer B, Harris TA, Ortner T. Sex and menstrual cycle influences on three aspects of attention. Physiol Behav. 2017;179:384-90.

25. Riley E, Okabe H, Germine L, Wilmer J, Esterman M, DeGutis J. Gender differences in sustained attentional control relate to gender inequality across countries. PLoS ONE. 2016;11:e0165100.

26. Bezdjian S, Baker LA, Lozano DI, Raine A. Assessing inattention and impulsivity in children during the Go/NoGo task. Br J Dev Psychol. 2009;27:365-83.

27. Conners CK, Epstein JN, Angold A, Klaric J. Continuous performance test performance in a normative epidemiological sample. J Abnorm Child Psychol. 2003;31:555-62.

28. Young JW, Light GA, Marston HM, Sharp R, Geyer MA. The 5-choice continuous performance test: evidence for a translational test of vigilance for mice. PLoS ONE. 2009;4:e4227.

29. Mirsky AF, Rosvold HE. The use of psychoactive drugs as a neuropsychological tool in studies of attention in man. In: Uhr I, Miller JG, editors. Drugs and behavior. New York, NY: Wiley; 1960. p. 375-92. .

30. Wang LJ, Huang YS, Chiang YL, Hsiao CC, Shang ZY, Chen CK. Clinical symptoms and performance on the continuous performance test in children with attention deficit hyperactivity disorder between subtypes: a natural follow-up study for 6 months. BMC Psychiatry. 2011;11:65.

31. Papaleo F, Erickson L, Liu G, Chen J, Weinberger DR. Effects of sex and COMT genotype on environmentally modulated cognitive control in mice. Proc Natl Acad Sci USA. 2012;109:20160-5.

32. Jentsch JD, Taylor JR. Sex-related differences in spatial divided attention and motor impulsivity in rats. Behav Neurosci. 2003;117:76-83.

33. Bayless DW, Darling JS, Stout WJ, Daniel JM. Sex differences in attentional processes in adult rats as measured by performance on the 5-choice serial reaction time task. Behav Brain Res. 2012;235:48-54.

34. Groves NJ, Burne TH. Sex-specific attentional deficits in adult vitamin D deficient BALB/c mice. Physiol Behav. 2016;157:94-101.

35. Grissom NM, Herdt CT, Desilets J, Lidsky-Everson J, Reyes TM. Dissociable deficits of executive function caused by gestational adversity are linked to specific transcriptional changes in the prefrontal cortex. Neuropsychopharmacology. 2015;40:1353-63.

36. Ciampoli M, Contarini G, Mereu M, Papaleo F. Attentional control in adolescent mice assessed with a modified five choice serial reaction time task. Sci Rep. 2017;7:9936.

37. Burton $\mathrm{CL}$, Fletcher PJ. Age and sex differences in impulsive action in rats: the role of dopamine and glutamate. Behav Brain Res. 2012;230:21-33.

38. Anshu K, Nair AK, Kumaresan UD, Kutty BM, Srinath S, Laxmi TR. Altered attentional processing in male and female rats in a prenatal valproic acid exposure model of autism spectrum disorder. Autism Res. 2017;10:1929-44. 
39. Lukkes JL, Thompson BS, Freund N, Andersen SL. The developmental interrelationships between activity, novelty preferences, and delay discounting in male and female rats. Dev Psychobiol. 2016;58:231-42.

40. Eckel CC, Grossman PJ. Men, women and risk aversion: experimental evidence. Handb Exp Econ Results. 2008;1:1061-73.

41. Orsini CA, Setlow B. Sex differences in animal models of decision making. J Neurosci Res. 2017;95:260-9.

42. van den Bos R, Homberg J, de Visser L. A critical review of sex differences in decision-making tasks: focus on the lowa Gambling Task. Behav Brain Res. 2013b;238:95-108.

43. Dunn BD, Dalgleish T, Lawrence AD. The somatic marker hypothesis: a critical evaluation. Neurosci Biobehav Rev. 2006;30:239-71.

44. Overman WH, Pierce A. lowa Gambling Task with non-clinical participants: effects of using real+virtual cards and additional trials. Front Psychol. 2013;4:935.

45. Singh V. Sex-differences, handedness, and lateralization in the lowa Gambling Task. Front Psychol. 2016;7:708.

46. Zhang F, Xiao L, Gu R. Does gender matter in the relationship between anxiety and decision-making? Front Psychol. 2017;8:2231.

47. Gruber KA, Callahan MF. ACTH-(4-10) through gamma-MSH: evidence for a new class of central autonomic nervous system-regulating peptides. Am J Physiol. 1989;257:R681-94.

48. van der Plas EA, Crone EA, van den Wildenberg WP, Tranel D, Bechara A. Executive control deficits in substance-dependent individuals: a comparison of alcohol, cocaine, and methamphetamine and of men and women. J Clin Exp Neuropsychol. 2009;31:706-19.

49. Kim HW, Kang Jl, Namkoong K, Jhung K, Ha RY, Kim SJ. Further evidence of a dissociation between decision-making under ambiguity and decisionmaking under risk in obsessive-compulsive disorder. J Affect Disord. 2015;176:118-24.

50. Overman W, Graham L, Redmond A, Eubank R, Boettcher L, Samplawski O, et al. Contemplation of moral dilemmas eliminates sex differences on the lowa gambling task. Behav Neurosci. 2006;120:817-25.

51. Starcke K, Agorku JD, Brand M. Exposure to unsolvable anagrams impairs performance on the lowa Gambling Task. Front Behav Neurosci. 2017;11:114.

52. van den Bos R, Davies W, Dellu-Hagedorn F, Goudriaan AE, Granon S, Homberg J, et al. Cross-species approaches to pathological gambling: a review targeting sex differences, adolescent vulnerability and ecological validity of research tools. Neurosci Biobehav Rev. 2013a;37:2454-71.

53. Dretsch MN, Tipples J. Sex differences moderate decision making behaviour in high impulsive sensation seekers. Cogn Emot. 2011;25:149-55.

54. Chiu YC, Lin $\mathrm{CH}$. Is deck $\mathrm{C}$ an advantageous deck in the lowa Gambling Task? Behav Brain Funct. 2007;3:37.

55. Lin $\mathrm{CH}$, Chiu YC, Lee PL, Hsieh JC. Is deck B a disadvantageous deck in the lowa Gambling Task? Behav Brain Funct. 2007;3:16

56. Addicott MA, Pearson JM, Wilson J, Platt ML, McClernon FJ. Smoking and the bandit: a preliminary study of smoker and nonsmoker differences in exploratory behavior measured with a multiarmed bandit task. Exp Clin Psychopharmacol. 2013;21:66-73.

57. Evans KL, Hampson E. Sex-dependent effects on tasks assessing reinforcement learning and interference inhibition. Front Psychol. 2015;6:1044.

58. Kovach CK, Daw ND, Rudrauf D, Tranel D, O'Doherty JP, Adolphs R. Anterior prefrontal cortex contributes to action selection through tracking of recent reward trends. J Neurosci. 2012;32:8434-42.

59. Derntl B, Pintzinger N, Kryspin-Exner I, Schopf V. The impact of sex hormone concentrations on decision-making in females and males. Front Neurosci. 2014;8:352.

60. Weafer J, De Arcangelis J, de Wit H. Sex differences in behavioral impulsivity in at-risk and non-risk drinkers. Front Psychiatry. 2015;6:72.

61. Cross CP, Copping LT, Campbell A. Sex differences in impulsivity: a metaanalysis. Psychol Bull. 2011;137:97-130.

62. Doi H, Nishitani S, Shinohara K. Sex difference in the relationship between salivary testosterone and inter-temporal choice. Horm Behav. 2015; 69:50-8.

63. Sidlauskaite J, Gonzalez-Madruga K, Smaragdi A, Riccelli R, Puzzo I, Batchelor M, et al. Sex differences in risk-based decision making in adolescents with conduct disorder. Eur Child Adolesc Psychiatry. 2017; doi: 10.1007/s00787-017-1024-9. [Epub ahead of print].

64. Heilbronner SR. Modeling risky decision-making in nonhuman animals: shared core features. Curr Opin Behav Sci. 2017;16:23-9.

65. van den Bos R, Jolles J, van der Knaap $L$, Baars A, de Visser L. Male and female Wistar rats differ in decision-making performance in a rodent version of the lowa Gambling Task. Behav Brain Res. 2012;234:375-9.

66. Orsini CA, Willis ML, Gilbert RJ, Bizon JL, Setlow B. Sex differences in a rat model of risky decision making. Behav Neurosci. 2016;130:50-61.
67. Peak JN, Turner KM, Burne TH. The effect of developmental vitamin D deficiency in male and female Sprague-Dawley rats on decision-making using a rodent gambling task. Physiol Behav. 2015;138:319-24.

68. Weston HI, Weston DD, Allen JL, Cory-Slechta DA. Sex-dependent impacts of low-level lead exposure and prenatal stress on impulsive choice behavior and associated biochemical and neurochemical manifestations. Neurotoxicology. 2014;44:169-83.

69. Brydges NM, Holmes MC, Harris AP, Cardinal RN, Hall J. Early life stress produces compulsive-like, but not impulsive, behavior in females. Behav Neurosci. 2015;129:300-8.

70. Eubig PA, Noe TE, Floresco SB, Sable JJ, Schantz SL. Sex differences in response to amphetamine in adult Long-Evans rats performing a delay-discounting task. Pharmacol Biochem Behav. 2014;118:1-9.

71. Chelonis JJ, Daniels-Shaw JL, Blake DJ, Paule MG. Developmental aspects of delayed matching-to-sample task performance in children. Neurotoxicol Teratol. 2000;22:683-94.

72. Leon I, Cimadevilla JM, Tascon L. Developmental gender differences in children in a virtual spatial memory task. Neuropsychology. 2014;28:485-95.

73. Loe IM, Luna B, Bledsoe IO, Yeom KW, Fritz BL, Feldman HM. Oculomotor assessments of executive function in preterm children. J Pediatr. 2012;161:427-33 e421.

74. Alarcon G, Cservenka A, Fair DA, Nagel BJ. Sex differences in the neural substrates of spatial working memory during adolescence are not mediated by endogenous testosterone. Brain Res. 2014;1593:40-54.

75. Castonguay N, Lussier M, Bugaiska A, Lord C, Bherer L. Executive functions in men and postmenopausal women. J Clin Exp Neuropsychol. 2015;37: 193-208.

76. Hsu HL, Chen DY, Tseng YC, Kuo YS, Huang YL, Chiu WT, et al. Sex differences in working memory after mild traumatic brain injury: a functional MR imaging study. Radiology. 2015;276:828-35.

77. Martoni RM, Salgari G, Galimberti E, Cavallini MC, O'Neill J. Effects of gender and executive function on visuospatial working memory in adult obsessivecompulsive disorder. Eur Arch Psychiatry Clin Neurosci. 2015;265:707-18.

78. McCarrey AC, An Y, Kitner-Triolo MH, Ferrucci L, Resnick SM. Sex differences in cognitive trajectories in clinically normal older adults. Psychol Aging. 2016;31:166-75.

79. Rahman $Q$, Abrahams $S$, Jussab F. Sex differences in a human analogue of the Radial Arm Maze: the "17-Box Maze Test". Brain Cogn. 2005;58:312-7.

80. Duff SJ, Hampson E. A sex difference on a novel spatial working memory task in humans. Brain Cogn. 2001;47:470-93.

81. Lejbak L, Vrbancic M, Crossley M. The female advantage in object location memory is robust to verbalizability and mode of presentation of test stimuli. Brain Cogn. 2009;69:148-53.

82. Reed JL, Gallagher NM, Sullivan M, Callicott JH, Green AE. Sex differences in verbal working memory performance emerge at very high loads of common neuroimaging tasks. Brain Cogn. 2017;113:56-64.

83. Voyer D, Voyer SD, Saint-Aubin J. Sex differences in visual-spatial working memory: a meta-analysis. Psychon Bull Rev. 2017;24:307-34.

84. Chai XJ, Jacobs LF. Effects of cue types on sex differences in human spatial memory. Behav Brain Res. 2010;208:336-42.

85. Seymoure $P$, Juraska JM. Sex differences in radial maze performance: influence of rearing environment and room cues. Psychobiology. 1996;24:33-7.

86. Gibbs RB, Johnson DA. Sex-specific effects of gonadectomy and hormone treatment on acquisition of a 12-arm radial maze task by Sprague Dawley rats. Endocrinology. 2008;149:3176-83.

87. Hall BJ, Abreu-Villaca Y, Cauley M, Junaid S, White H, Kiany A, et al. The ventral hippocampal muscarinic cholinergic system plays a key role in sexual dimorphisms of spatial working memory in rats. Neuropharmacology. 2017;117:106-13.

88. Harris JC, Martinez JM, Grozdanov PN, Bergeson SE, Grammas P, MacDonald CC. The Cstf2t polyadenylation gene plays a sex-specific role in learning behaviors in mice. PLoS ONE. 2016;11:e0165976.

89. Luine V, Gomez J, Beck K, Bowman R. Sex differences in chronic stress effects on cognition in rodents. Pharmacol Biochem Behav. 2017;152:13-9.

90. Bimonte HA, Denenberg VH. Sex differences in vicarious trial-and-error behavior during radial arm maze learning. Physiol Behav. 2000;68:495-9.

91. West RK, Maynard ME, Leasure JL. Binge ethanol effects on prefrontal cortex neurons, spatial working memory and task-induced neuronal activation in male and female rats. Physiol Behav. 2018;188:79-85.

92. Sutcliffe JS, Marshall KM, Neill JC. Influence of gender on working and spatial memory in the novel object recognition task in the rat. Behav Brain Res. 2007;177:117-25.

93. Roddick KM, Schellinck HM, Brown RE. Olfactory delayed matching to sample performance in mice: sex differences in the 5XFAD mouse model of Alzheimer's disease. Behav Brain Res. 2014;270:165-70. 
94. Rodriguez JS, Zurcher NR, Bartlett TQ, Nathanielsz PW, Nijland MJ. CANTAB delayed matching to sample task performance in juvenile baboons. J Neurosci Methods. 2011a;196:258-63.

95. Grilly DM. Sex differences in delayed matching-to-sample performance of chimpanzees. Psychol Rep. 1975;37:203-7.

96. Fridberg DJ, Gerst KR, Finn PR. Effects of working memory load, a history of conduct disorder, and sex on decision making in substance dependent individuals. Drug Alcohol Depend. 2013;133:654-60.

97. Nooner KB, Hooper SR, De Bellis MD. An examination of sex differences on neurocognitive functioning and behavior problems in maltreated youth. Psychol Trauma. 2017; 10:435-443.

98. Lee J, Smith JP. Regional disparities in adult height, educational attainment and gender difference in late-life cognition: findings from the Longitudinal Aging Study in India (LASI). J Econ Ageing. 2014;4:26-34.

99. Lei X, Hu Y, McArdle JJ, Smith JP, Zhao Y. Gender differences in cognition among older adults in China. J Hum Resour. 2012;47:951-71.

100. Lei $X$, Smith JP, Sun X, Zhao Y. Gender differences in cognition in China and reasons for change over time: evidence from CHARLS. J Econ Ageing. 2014:4:46-55.

101. Pereira VH, Costa PS, Santos NC, Cunha PG, Correia-Neves M, Palha JA, et al. Adult body height is a good predictor of different dimensions of cognitive function in aged individuals: a cross-sectional study. Front Aging Neurosci. 2016;8:217

102. Shim SY, Cho SJ, Kong KA, Park EA. Gestational age-specific sex difference in mortality and morbidities of preterm infants: a nationwide study. Sci Rep. 2017;7:6161.

103. Skuse $\mathrm{DH}$. Imprinting, the $\mathrm{X}$-chromosome, and the male brain: explaining sex differences in the liability to autism. Pediatr Res. 2000;47:9-16.

104. Vu HD, Dickinson C, Kandasamy Y. Sex difference in mortality for premature and low birth weight neonates: a systematic review. Am J Perinatol. 2017; 35:707715.

105. Davis EP, Pfaff D. Sexually dimorphic responses to early adversity: implications for affective problems and autism spectrum disorder. Psychoneuroendocrinology. 2014;49:11-25.

106. McCarthy MM. Sex differences in the developing brain as a source of inherent risk. Dialog- Clin Neurosci. 2016;18:361-72.

107. Karemaker R, Heijnen CJ, Veen S, Baerts W, Samsom J, Visser GH, et al. Differences in behavioral outcome and motor development at school age after neonatal treatment for chronic lung disease with dexamethasone versus hydrocortisone. Pediatr Res. 2006;60:745-50.

108. Rodriguez JS, Zurcher NR, Keenan KE, Bartlett TQ, Nathanielsz PW, Nijland MJ. Prenatal betamethasone exposure has sex specific effects in reversal learning and attention in juvenile baboons. Am J Obstet Gynecol. 2011b;204:545 e541-10.

109. Rodriguez JS, Bartlett TQ, Keenan KE, Nathanielsz PW, Nijland MJ. Sexdependent cognitive performance in baboon offspring following maternal caloric restriction in pregnancy and lactation. Reprod Sci. 2012;19:493-504.

110. Lloyd $S A$, Oltean $C$, Pass $H$, Phillips $B$, Staton $K$, Robertson $C L$, et al. Prenatal exposure to psychostimulants increases impulsivity, compulsivity, and motivation for rewards in adult mice. Physiol Behav. 2013;119:43-51.

111. Talge NM, Allswede DM, Holzman C. Gestational age at term, delivery circumstance, and their association with childhood attention deficit hyperactivity disorder symptoms. Paediatr Perinat Epidemiol. 2016;30:171-80.

112. Melchior M, Hersi R, van der Waerden J, Larroque B, Saurel-Cubizolles MJ, Chollet $A$, et al. Maternal tobacco smoking in pregnancy and children's socioemotional development at age 5: the EDEN mother-child birth cohort study. Eur Psychiatry. 2015:30:562-8.

113. Kobrosly RW, Evans S, Miodovnik A, Barrett ES, Thurston SW, Calafat AM, et al. Prenatal phthalate exposures and neurobehavioral development scores in boys and girls at 6-10 years of age. Environ Health Perspect. 2014;122:521-8.

114. Hehar H, Yeates K, Kolb B, Esser MJ, Mychasiuk R. Impulsivity and concussion in juvenile rats: examining molecular and structural aspects of the frontostriatal pathway. PLoS ONE. 2015;10:e0139842.

115. Wallensteen L, Zimmermann M, Thomsen Sandberg M, Gezelius A, Nordenstrom A, Hirvikoski T, et al. Sex-dimorphic effects of prenatal treatment with dexamethasone. J Clin Endocrinol Metab. 2016;101:3838-46.

116. Andersen HR, Debes F, Wohlfahrt-Veje C, Murata K, Grandjean P. Occupational pesticide exposure in early pregnancy associated with sex-specific neurobehavioral deficits in the children at school age. Neurotoxicol Teratol. 2015:47:1-9.

117. Loi M, Mossink JC, Meerhoff GF, Den Blaauwen JL, Lucassen PJ, Joels M. Effects of early-life stress on cognitive function and hippocampal structure in female rodents. Neuroscience. 2017;342:101-19.

118. Selleck RA, Lake C, Estrada V, Riederer J, Andrzejewski M, Sadeghian K, et al. Endogenous opioid signaling in the medial prefrontal cortex is required for the expression of hunger-induced impulsive action. Neuropsychopharmacology. 2015;40:2464-74

119. Feja $M$, Koch $M$. Ventral medial prefrontal cortex inactivation impairs impulse control but does not affect delay-discounting in rats. Behav Brain Res. 2014;264:230-9.

120. Jupp B, Caprioli D, Saigal N, Reverte I, Shrestha S, Cumming P, et al. Dopaminergic and GABA-ergic markers of impulsivity in rats: evidence for anatomical localisation in ventral striatum and prefrontal cortex. Eur J Neurosci. 2013;37:1519-28.

121. Tessitore A, Santangelo G, De Micco R, Vitale C, Giordano A, Raimo S, et al. Cortical thickness changes in patients with Parkinson's disease and impulse control disorders. Parkinsonism Relat Disord. 2016; 24:119-25.

122. Yates JR, Darna M, Beckmann JS, Dwoskin LP, Bardo MT. Individual differences in impulsive action and dopamine transporter function in rat orbitofrontal cortex. Neuroscience. 2016;313:122-9.

123. Boy $F$, Evans $C J$, Edden RA, Lawrence AD, Singh KD, Husain $M$, et al. Dorsolateral prefrontal gamma-aminobutyric acid in men predicts individual differences in rash impulsivity. Biol Psychiatry. 2011;70:866-72.

124. Murphy ER, Fernando AB, Urcelay GP, Robinson ES, Mar AC, Theobald DE, et al. Impulsive behaviour induced by both NMDA receptor antagonism and GABAA receptor activation in rat ventromedial prefrontal cortex. Psychopharmacol (Berl). 2012;219:401-10.

125. Benn A, Robinson ES. Investigating glutamatergic mechanism in attention and impulse control using rats in a modified 5-choice serial reaction time task. PLoS ONE. 2014;9:e115374.

126. Logue SF, Gould TJ. The neural and genetic basis of executive function: attention, cognitive flexibility, and response inhibition. Pharmacol Biochem Behav. 2014;123:45-54.

127. Sanchez-Roige S, Ripley TL, Stephens DN. Alleviating waiting impulsivity and perseverative responding by mu-opioid receptor antagonism in two inbred mouse strains. Psychopharmacol (Berl). 2015;232:1483-92.

128. D'Amour-Horvat V, Leyton M. Impulsive actions and choices in laboratory animals and humans: effects of high vs. low dopamine states produced by systemic treatments given to neurologically intact subjects. Front Behav Neurosci. 2014;8:432.

129. Kolisnyk B, Al-Onaizi MA, Hirata PH, Guzman MS, Nikolova S, Barbash S, et al. Forebrain deletion of the vesicular acetylcholine transporter results in deficits in executive function, metabolic, and RNA splicing abnormalities in the prefrontal cortex. J Neurosci. 2013;33:14908-20.

130. Staiti AM, Morgane PJ, Galler JR, Grivetti JY, Bass DC, Mokler DJ. A microdialysis study of the medial prefrontal cortex of adolescent and adult rats. Neuropharmacology. 2011;61:544-9.

131. Alves NC, Bailey CD, Nashmi R, Lambe EK. Developmental sex differences in nicotinic currents of prefrontal layer VI neurons in mice and rats. PLoS ONE. 2010;5:e9261

132. Andersen SL, Teicher MH. Sex differences in dopamine receptors and their relevance to ADHD. Neurosci Biobehav Rev. 2000;24:137-41.

133. Gurvich C, Rossell SL. Dopamine and cognitive control: sex-by-genotype interactions influence the capacity to switch attention. Behav Brain Res. 2015;281:96-101.

134. LaRoche RB, Morgan RE. Adolescent fluoxetine exposure produces enduring, sex-specific alterations of visual discrimination and attention in rats. Neurotoxicol Teratol. 2007;29:96-107.

135. Kolb B, Mychasiuk R, Muhammad A, Li Y, Frost DO, Gibb R. Experience and the developing prefrontal cortex. Proc Natl Acad Sci USA. 2012;109:17186-93.

136. Rakic P, Bourgeois JP, Eckenhoff MF, Zecevic N, Goldman-Rakic PS. Concurrent overproduction of synapses in diverse regions of the primate cerebral cortex. Science. 1986;232:232-5.

137. Samaco RC, McGraw CM, Ward CS, Sun Y, Neul JL, Zoghbi HY. Female Mecp2+/mice display robust behavioral deficits on two different genetic backgrounds providing a framework for pre-clinical studies. Hum Mol Genet. 2013; 22:96-109.

138. Volk L, Chiu SL, Sharma K, Huganir RL. Glutamate synapses in human cognitive disorders. Annu Rev Neurosci. 2015;38:127-49.

139. Wu EQ, Shi L, Birnbaum H, Hudson T, Kessler R. Annual prevalence of diagnosed schizophrenia in the USA: a claims data analysis approach. Psychol Med. 2006;36:1535-40.

140. Stevens B, Allen NJ, Vazquez LE, Howell GR, Christopherson KS, Nouri N, et al. The classical complement cascade mediates CNS synapse elimination. Cell. 2007;131:1164-78.

141. Schwarz JM, Sholar PW, Bilbo SD. Sex differences in microglial colonization of the developing rat brain. J Neurochem. 2012;120:948-63.

142. Hanamsagar R, Alter MD, Block CS, Sullivan H, Bolton JL, Bilbo SD. Generation of a microglial developmental index in mice and in humans reveals a sex difference in maturation and immune reactivity. Glia. 2017;65:1504-20. 
Let's call the whole thing off: evaluating gender and sex differences in... NM Grissom and TM Reyes

143. Rahimian R, Cordeau P, Jr., Kriz J. Brain response to injuries: When microglia go sexist. Neuroscience. 2018; doi: 10.1016/j.neuroscience.2018.02.048. [Epub ahead of print].

144. Bollinger JL, Bergeon Burns CM, Wellman CL. Differential effects of stress on microglial cell activation in male and female medial prefrontal cortex. Brain Behav Immun. 2016;52:88-97.

145. Bollinger JL, Collins KE, Patel R, Wellman CL. Behavioral stress alters corticolimbic microglia in a sex- and brain region-specific manner. PLoS ONE. 2017;12: e0187631.

146. Barton EA, Baker C, Leasure JL. Investigation of sex differences in the microglial response to binge ethanol and exercise. Brain Sci. 2017; 7: 139. https://doi.org/ 10.3390/brainsci7100139
147. Grissom NM, McKee SE, Schoch H, Bowman N, Havekes R, O'Brien WT, et al. Male-specific deficits in natural reward learning in a mouse model of neurodevelopmental disorders. Mol Psychiatry. 2018;23:544-55.

148. Reber J, Tranel D. Sex differences in the functional lateralization of emotion and decision making in the human brain. J Neurosci Res. 2017;95:270-8.

149. Sutterer MJ, Koscik TR, Tranel D. Sex-related functional asymmetry of the ventromedial prefrontal cortex in regard to decision-making under risk and ambiguity. Neuropsychologia. 2015;75:265-73.

150. Tranel D, Bechara A. Sex-related functional asymmetry of the amygdala: preliminary evidence using a case-matched lesion approach. Neurocase. 2009;15:217-34.

151. Tranel D, Damasio H, Denburg NL, Bechara A. Does gender play a role in functional asymmetry of ventromedial prefrontal cortex? Brain. 2005;128:2872-81. 